\title{
ERS: LATENT DIRICHLET ALLOCATION BASED E-COMMERCE RECOMMENDATION SYSTEM USING DEEP NEURAL NETWORK
}

\section{R.Preethi, Research scholar,}

Dept. of Computer Science, Karpagam Academy of Higher Education

Dr.S. Sheeja, Professor,

Dept. of Computer Science, Karpagam Academy of Higher Education

\begin{abstract}
Online shopping is the best solution for busy life in today's world. In the past decade, there had been a massive change in the way of customer's shopping. In days individuals can help by keeping their time and effort by purchasing pieces online with various websites. Their particular working experience using a system is present in reviews/feedback given for these products. Recommender's solutions offer heart-felt selections so that people can end-users just by recording their valuable pursuits together with preferences. Recommender devices provide fantastic opportunities to organizations; therefore, establishing new recommender system strategies and strategies has been increasing the focus. In this document, the ERS (Ecommerce recommendation system) using a serious neural community is recommended. We tend to produce some glossary about capabilities from each product or service kind and examine these to get rid of unimportant phrases by using Latent Dirichlet Allocation. Afterward, people being used any full nerve system network having the capacity to extract rich features inside reviews-characteristics matrix to deal with sparsity, ambiguity, apart from redundancy. Most people have applied factorization because of the collaborative, integrated choice supply regulations.
\end{abstract}

Keywords:

LDA, ERS, DNN, Recommendation, Ecommerce, Neural Network

Article Received: 18 October 2020, Revised: 3 November 2020, Accepted: 24 December 2020

\section{INTRODUCTION}

In the busy world, millennials like the web sales/purchases to save their interval and money. Besides, this data is normally huge throughout mother nature due to the accessibility of products/items in big amounts with their grammar offered by providers, capped by using buyers comments available as evaluations and also critical opinions[1]. Ratings grow to be reflections linked with the users endorsement based on the latest scale (say, 0-5).Earlier studies are likely to be exact first hand information about the own personal user's sensible knowledge on product/service. Evaluations along with testimonials indicate a good buyer's achievement [2]. The sooner job in regards to investigation during the situation with wording typically points out the thought of what actually message exploration is actually happened. sometimes employing a lexicon-based process or possibly applying unit mastering approaches is recommended. It can give you the polarity good type of ensuing while it is beneficial or maybe damaging as well as simple. Considering a small portion of such specifics seems to be improper,so increase the value of automatic recommender systems with the capability to encourage relevant merchandise and brand-new items to the shoppers. Besides, change and transform for people in addition to delivering guidelines on the everincreasing data is vital and challenging problem intended for online maintenance shops for instance e-learning[5]. Recommender models can be a subset associated with blocking models that try and estimate users' personal preferences for the piece and provide unique recommendation influenced at this time of examination for a end-user plus feel [6]. Frauds could grab the wearer's bank account on websites to work with the ex balance or simply 
work with a compromised or perhaps artificial MasterCard to subscribe the latest affirmation. Sometimes, event reasons reduce the internet site and its particular people. As a result, pricey emergency to develop powerful fraudulence discovery products to halt this sort of habits [7]. The recommended method helps to remove literal and aesthetic features via users' evaluations, which have been graded by every user, and merges these people into important features dependent on canonical connection analysis

Nowadays, deep sensory networks show promising brings into reality in the field for recommender's products where nonlinear functions bring learning intricate relations amongst the data. In this paper, most people attempt to present insights about Deep Neural Network strategies for RS and use the full features of the performance associated with Ecommerce Professional's recommendation. The rest of this kind of papers are organized in the following. Section II presents the very literature review and connected works. Sections III looks at the problem outline and the planned approach. Spot IV supplies information about the details preprocessing and even analyzes the consequences of the treatment plan analysis. in section $\mathrm{V}$, we determine the current study, its limits, and foreseeable future research information.

\section{BACKGROUND OF THE STUDY}

Many studies try to combine customer published reviews throughout generating regulations. Bi, J.-W. et al [1] A deep neural network-based professional recommendation algorithm is commonly proposed exactly where common user rating, end-user basic, object basic facts, and average garment report are used. The recommendation criteria' central core is to make a regression style for predictive prophetic user evaluations based on nerve organ networks. Chehal, D. et al [2] The advice system's real success depends on generating suggestions swiftly and directed in regards to the right buyers. This system of activity should be because fundamental because it is striking a good interactive discussion between 2 different people. A number of RS kinds have been discovered over the years based on different methods as covered in this fictional works. RS determined user ratings offer a good sharp edge around some other RS. It is about with real life connections used by their customers, efficiently implicit as well as explicit factors to generate a good deal with much better recommendations. Jannach, D. et al [3] The particular systematic examination of attributes in productive real-world advice, which has not been worn out the commerce enligne domain ahead as far as we have known. Often the gathered information helped people and electrical industrial engineer to give, new remedies that check out various signals in sychronized and increased conjecture reliability compared with prior tactics. The exact esprit involving particular capabilities to the conjecture process could be domain-specific. The reccommended homework method is general and can be utilized in various other websites simultaneously. The very creators determined the qualities that the professionals used in the current analyses They depend upon observations connected with practical strategies and in accordance with general worries regarding to specific niche market categories' practices on the studying

literature. Wang, S. et al [7] Frauds are generally intrinsically authoritarian analysis because they are engineered to stop detection. The good news is that the editors can view millions of deals per day, thereby accumulating plenty of fraud selections to train a very detailed RNN (recurrent neural network) version that conveys the precise, detailed just click information and the complete sequences. Using proper coping with unbalanced mastering, strategy float, and current offering troubles are recommended.The consultants present typical characteristics in addition to the outwardly specific 
plus high-priced that will figure out, truly size to supplement the order volumes an authors receive while having accuracy and even reliability by no means reached by simply classic approaches based upon get worse options. In addition, this approach is easy, without abnormal ad hoc factor engineering, displaying another benefit can be linked by using RNN.

Kawashima, T., et al [8] Suggested the latest assessment prediction technique, which often utilizes ordinal regression determined by LDA using multimodal benefits. The precise authors can easily see the functionality enhancement belonging to the ranking auguration by utilizing multimodal functions from the plan for treatment results bought by frequently applying the actual proposed method of the precise information set.The problem concerning determining the best number of canonical variants continues to be unsolved. Zhang, D. et al [9] Designed a knowledge to give learning course of action that engages deep sensation problems in marketing networks towards estimate LDA. Final results about contract category responsibilities present a straightforward DNN could close LDA quite nicely, while inference will drastically improve speed. This kind of preliminary research uncovers that transitioning knowledge from Bayesian merchandise to physical models is possible.

\section{E-COMMERCE RECOMMENDER} SYSTEM USING DNN

Provided three types of testimonials, we have figured out the typical rankings and good support scores along with each summary category. Reviewers frequently assign a more massive average position score.

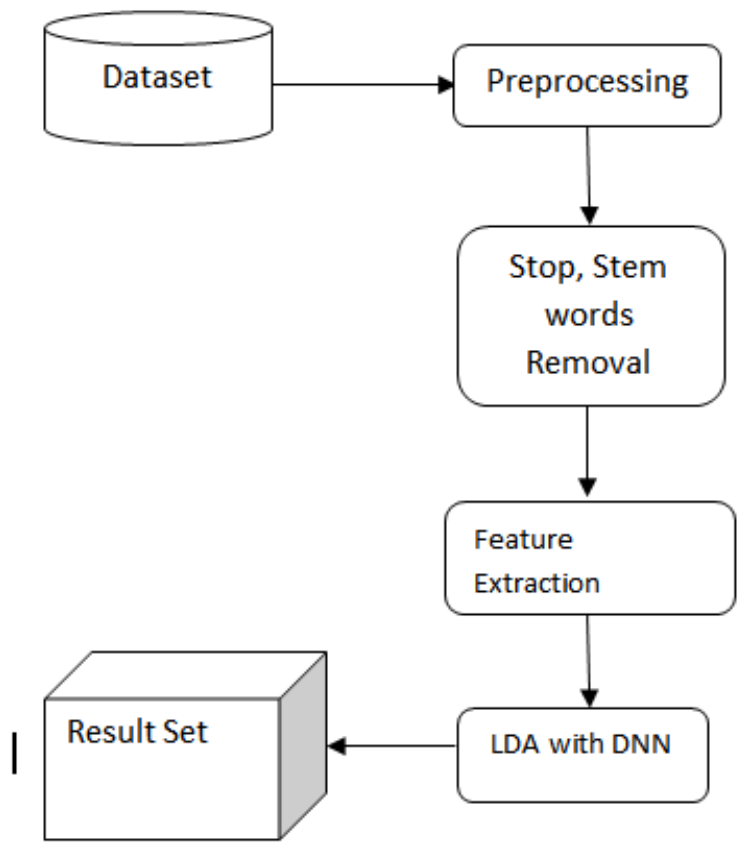

Figure 1: System Architecture

Most of us compare a typical rating for that reason thousands of assessments are done through evaluation.. It is experienced that premature evaluations usually tend to keep enterprises with a more significant standing review than persons from several other two types. Remember that in the Amazon, Yelp dataset possesses each. It all depends ballots on opinions; people use the amount associated with Absolutely yes polls to symbolize the particular useful assistance and quite a few good reviews. In the Yelp dataset, we take advantage of the number of Effective votes which help the article. 


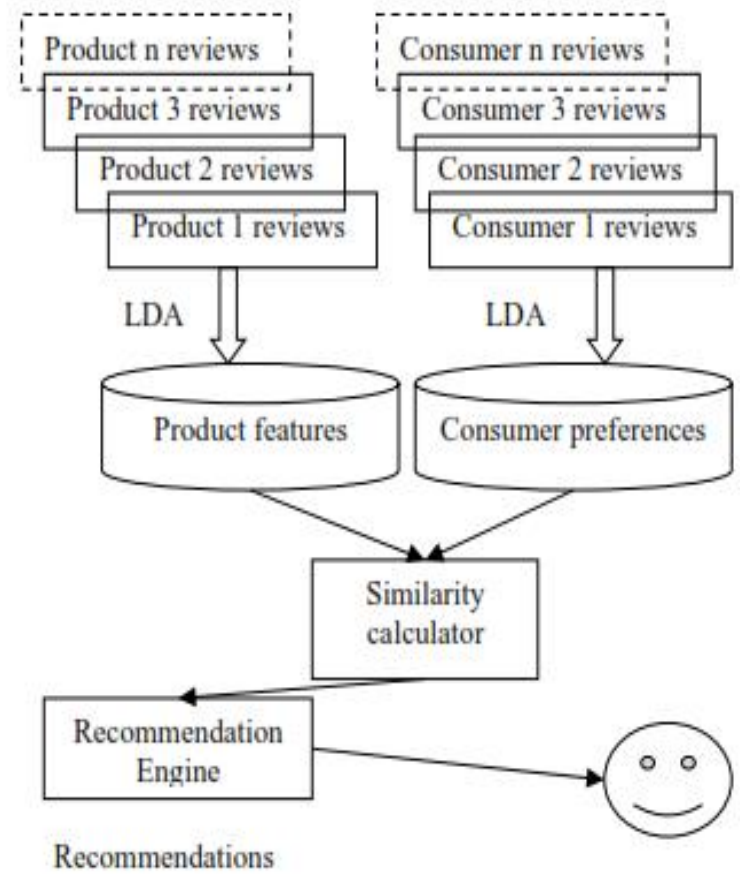

Figure 2: Recommendation Engine using LDA

\subsection{Data Cleaning}

The information cleanup includes a couple of primary methods, the following a few.

\subsection{Preprocessing}

Most of us first eliminate reviews from anonymous men and women since we wish to connect every analysis using a particular customer. Most of us then take out copy ratings, usually due to the identical product or service editions. We all furthermore take away less productive users besides unwanted items: we keep the customers who have distributed at least a few and several evaluations, plus goods that may have obtained at the very least five and even all 5 assessments inside the Amazon web Yelp datasets correspondingly. We all take out end words and phrases and phrases and extremely irregular words and phrases regarding examine text messages.

\subsection{Stemming Removing}

Our emphasis is to analyze the first rehoming actions connected with real The amazon.com online market place and Yelp users.
Nevertheless, as verified in [3], the amount of spam crucial reviews offers more and more developed ecommerce websites and discovered that will, regarding $10 \%$ to $15 \%$ associated with testimonials, echoed before testimonials. It may become placed through critique junk emailers. It will be possible that junk evaluations are usually published to offer prejudiced or maybe fake thoughts on several products to influence the precise consumers' belief of the options which not directly inflating and damaging the product's position. The existence of unrequested mail opinions can result in incorrect results in our examination. For that reason, we must eliminate the evaluation of junk emailers within our information cleaning technique.

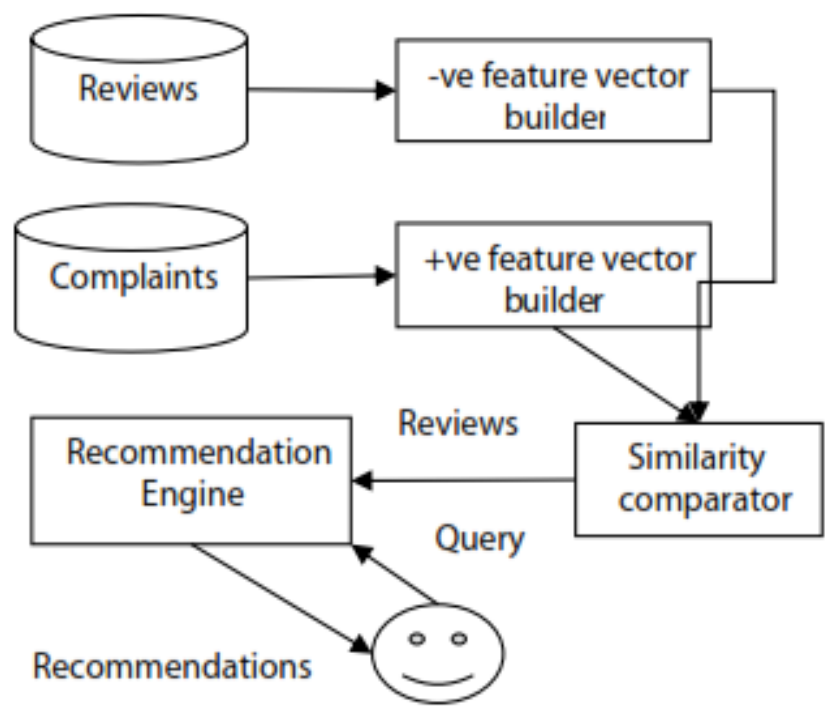

Figure 3: Similarity Comparator in the Recommendation engine

\subsection{E-PREDICT}

An initial disadvantage of the precise presented heuristics-based techniques is usually that the reranking strategy is coarse-grained. A lot of finetuning is required to find a good combination of heuristics and then complementing weights whilst hybrids are applied in the process. We are thinking about making a learning-based approach which is capable of thinking about a selection of indicators inside simultaneous and qualified to determine 
perfect weights instantly. The group activity's final results are data scores which will express the probability of the consumer which may click on and buy an endorsed thing. As opposed to standard class difficulties, all of us usually do not use these types of results to identify the main good-quality things. Nevertheless, we make use of them to obtain ranking products identified as attainable recommendations through the baseline project. Overall, the outcomes demonstrate which reranking the actual advice through contemplating a number of different session-based components can lead to significant accuracy earnings. The actual studies supply additional facts that will give modern-day heavy understanding methods which can help to acquire more substantial results in this site than we are able to achieve along with additional conventional strategies such as random woodlands or perhaps together with personally updated excess weight aspects.

\section{Mathematical Model:}

Let $\mathrm{S}$ is the Whole System Consist of

$\mathrm{S}=\{\mathrm{I}, \mathrm{P}, \mathrm{O}\}$

$\mathrm{I}=$ Input.

$\mathrm{I}=\{\mathrm{SU}, \mathrm{EU}, \mathrm{A}, \mathrm{C}, \mathrm{D}\}$

$\mathrm{SU}=$ Social Network Users

$\mathrm{SU}=\{\mathrm{su} 1, \mathrm{su} 2 \ldots \mathrm{sun}\}$

$\mathrm{EU}=\{$ eu1, eu2 ... eun $\}$

$\mathrm{A}=\mathrm{E}$ Commerce Advertisers

$\mathrm{A}=\{\mathrm{a} 1, \mathrm{a} 2, \ldots, \mathrm{an}\}$

$\mathrm{C}=$ Content of product Profile in Network.

$\mathrm{C}=\{\mathrm{c} 1, \mathrm{c} 2, \mathrm{c} 3 \ldots \mathrm{cn}\}$

$\mathrm{D}=$ Dataset

$\mathrm{P}=$ Process:

1) Step1: Marketer will publish the product within the E-commerce website.

2) Step2: Social Networks customers will be observed on Interpersonal sites wherever the user may view, discuss, and give remarks on the posts. The consumer can deliver and get friend demand in social networking.
3) Step3: Social Network site subsequent attribute is utilized to suggest product upon E-commerce site

\subsection{Latent Dirichlet Allocation (LDA)}

Latent Dirichlet Allocation (LDA) is among the most widespread. It will help get each direct and acted subjects, such as relative's phrases for every issue. The whole course of action is launched on Bayesian systems. Take an assortment of seller or even buyer's reviews, $R=\left\{r_{1}, r_{2}, \ldots, r_{\mathrm{r}}\right\}$. Each review, call it $r$, is represented as a collection of words $r=\left\{w_{1 r}\right.$, $\left.w_{2 r}, \ldots, w_{N r}\right\}$, here $r_{R}$ denotes the $|R|^{\text {th }}$ review in the collection $\mathrm{R}$, and $w_{N r}$ denotes the Nth word in review $r$.

The latent topics are denoted by $K=\left\{\Phi_{1}\right.$, $\left.\Phi_{2}, \ldots, \Phi_{K}\right\}$, where $|K|$ is the topic number.

Typically the LDA product's essential basic principle is allocating words by way of multiple, specific reviews to many new, simple document, deciding a possibility with each term. Subsequently, every brand-new record presents a subject including a few highly-related co-occurring phrases (i. age., best info). The actual generative procedure for every single brand's first document is usually described as uses:

1. For each review, choose the topic distribution $\theta r \sim \operatorname{Dirichlet}(\alpha)$

2. For each word $w_{N d}$ in the review $r$

(a) Choose a topic $z_{N r} \sim$ Multinomial $(\theta r)$

(b) Choose a word $w_{N r}$ from $p\left(w_{N r} \mid z_{N r}, \beta\right)$ (i.e., $\left.\Phi_{r, N}\right)$ IV. DISCUSSION

Needs the effectiveness of the particular recommendation method, we have used datasets coming from Amazon in addition to Yelp datasets. The number of components of a good advice listing must be memory joggers plus that approach is needed to pick the simple recommendations largely depends on the cyberspace particulars or even program. Therefore, the trouble is available to discover a stability involving the suggestion 
regarding to currently identifies products and also even for booking recommendations are used.

Table 1: Algorithm Comparison

\begin{tabular}{|l|c|c|c|}
\hline \multicolumn{1}{|c|}{$\begin{array}{c}\text { Algorith } \\
m\end{array}$} & $\begin{array}{l}\text { Time in } \\
\text { Ms }\end{array}$ & $\begin{array}{l}\text { No of } \\
\text { produces }\end{array}$ & $\begin{array}{l}\text { Accura } \\
\text { cy }\end{array}$ \\
\hline LDA & 2 & 1 & $60 \%$ \\
& & 1 & $30 \%$ \\
\hline $\begin{array}{l}\text { Sentimental } \\
\text { Analysis }\end{array}$ & 6 & 2 & \\
\hline Neural & 3 & 1 & $70 \%$ \\
Network & & 0 & \\
\hline LDA with & 1 & 2 & $89 \%$ \\
DNN & $\cdot$ & 0 & \\
& 8 & & \\
& 5 & & \\
\hline
\end{tabular}

Table 1 illustrates the different algorithms comparing Time, Accuracy, and total no of products.

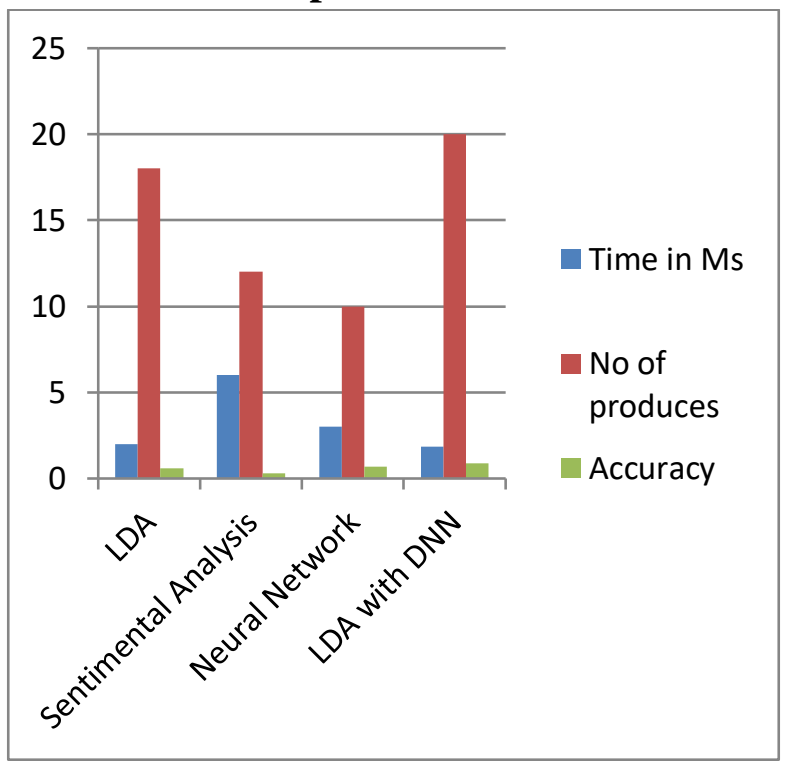

For Example, An individual can add pals, communicate with good friends, etc. Alternatively, the product $\mathrm{DB}$ is served by the admin, which includes the entire report on products to get listed. Admin is typically given the privilege to incorporate the products from the database and erase your database's work. Every product inside the database is usually mapped into a unique designation as a Product or service ID. Anyone's embedding and also product embedding are done to interlink the repository. The user, while logs into the system to get the data,he/she initially can view the number of products detailed as per the needs. Once the customer makes his or her visit to the product or service, it is the initial visit in the user for the specific product or service. The recommended system is developed on a surface rule The advice system Operates without often knowing the historical obtain records. Therefore the trips are saved as the insight for promoting products. The product or service catalog is displayed with the entire Product Big name, Brand Name, Treasure, Image, Has a high ranking, and Buy alternative.

\section{CONCLUSION}

The purpose of the papers was to recommend, implement, and experimentally assess deep neural network structures as distinguish products in ebusiness[10] data utilizing LDA. The device is used for suggesting products coming from e-commerce sites to tiny blogging customers who do not need history of buying records. Although there is not much performance obtained with and without functions, the use of various sets of part information, hyper parameters, and regularization methods can be considered for dealing with the chilly start issue and enhancing the overall performance of the product. This can slow up the testing mistake and strengthen the design further through improving the courses, reducing the main between coaching and affirmation loss, which may be monitored with the training chart. Thus, all of us conclude that people have offered a suggestion system for conquering the existing trouble.

\section{REFERENCES}

[1] Bi, J.-W., Liu, Y., \& Fan, Z.-P. (2019). A deep neural networks based recommendation algorithm using user and item basic data. International Journal of Machine Learning and 
Cybernetics. doi:10.1007/s13042-019-00981$\mathrm{y}$

[2] Chehal, D., Gupta, P., \& Gulati, P. (2020). Implementation and comparison of topic modeling techniques based on user reviews in e-commerce recommendations. Journal of Ambient Intelligence and Humanized Computing. doi:10.1007/s12652020-01956-6

[3] Jannach, D., Ludewig, M., \&Lerche, L. (2017). Session-based item recommendation in e-commerce: on short-term intents, reminders, trends and discounts. User Modeling and User-Adapted Interaction, 27(35), 351-392. doi:10.1007/s11257-017-9194-1

[4] Islam, C. S., \&Alauddin, M. (2018). A Novel Idea of Classification of E-commerce Products Using Deep Convolutional Neural Network. 2018 4th International Conference on Electrical Engineering and Information \& Communication Technology (iCEEiCT). doi:10.1109/ceeict.2018.8628161

[5] Syamala, M., \&Nalini, N. J. (2019). LDA and Deep Learning: A Combined Approach for Feature Extraction and Sentiment Analysis. 2019 10th International Conference on Computing, Communication and Networking Technologies

(ICCCNT). doi:10.1109/iccent45670.2019.89 44823

[6] Shoja, B. M., \&Tabrizi, N. (2019). Customer Reviews Analysis with Deep Neural Networks for E-Commerce Recommender Systems. IEEE Access, 11. doi:10.1109/access.2019.2937518

[7] Wang, S., Liu, C., Gao, X., Qu, H., \& Xu, W. (2017). Session-Based Fraud Detection in Online E-Commerce Transactions Using Recurrent Neural Networks. Lecture Notes in Computer Science, 241252. doi:10.1007/978-3-319-71273-4_20
[8] Kawashima, T., Ogawa, T., \&Haseyama, M. (2013). A rating prediction method for ecommerce application using ordinal regression based on LDA with multimodal features. 2013 IEEE 2nd Global Conference on Consumer Electronics

(GCCE). doi:10.1109/gcce.2013.6664818

[9] Zhang, D., Luo, T., \& Wang, D. (2016). Learning from LDA Using Deep Neural Networks. Lecture Notes in Computer Science, 657-664. doi:10.1007/978-3-31950496-4_59

[10] Dr.S.Sheeja, Preethi.Current trendsand tools of sentiment analysis and opinion mining,international journal of future generation communication and networking,2020,vol.13,issue3. 